\title{
Psychometric evaluation of the anticipatory grief scale in a sample of family caregivers in the context of palliative care
}

\author{
Maja Holm ${ }^{1 *}$ (D) Anette Alvariza ${ }^{2,3}$, Carl-Johan Fürst ${ }^{4}$, Joakim Öhlen ${ }^{5,6}$ and Kristofer Årestedt ${ }^{7,8}$
}

\begin{abstract}
Introduction: In palliative care, family caregivers are often faced with experiences of grief in anticipation of the loss of a close person. An instrument designed to measure this form of grief is the Anticipatory Grief Scale, which includes 27 items and has been used in several studies in various contexts. However, the instrument has not been validated.

Aim: The aim was to evaluate the psychometric properties, focusing on the factor structure, of the Anticipatory Grief Scale in a sample of family caregivers in palliative care.

Methods: The study had a cross-sectional design. Data were collected from an intervention study in palliative home care that took place between 2013 and 2014. In total, 270 family caregivers in palliative care completed a baseline questionnaire, including the Anticipatory Grief Scale. The factor structure of the scale was evaluated using exploratory factor analysis.

Results: The initial factor analysis suggested a four-factor solution, but, due to weak communalities, extensive crossloadings, and item inconsistencies, the model was problematic. Further analysis supported that the scale should be reduced to 13 items and two factors. The two subscales captured the behavioral and emotional reactions of grief in family caregivers in palliative care and were named Behavioral reactions and Emotional reactions. This modified version will hereafter be named AGS-13.
\end{abstract}

Conclusions: This validation study of the Anticipatory Grief Scale resulted in a revised two-factor model, AGS-13, that appears to be promising for use in palliative care but needs to be tested further.

Keywords: Anticipatory grief, Palliative care, Family caregivers, Instrument development, Factor analysis, Nursing

\section{Background}

Grief is generally defined as the psychological and physiological response to the death of a close person [1]. Grief is, in itself, not pathological, but the reactions and consequences of it can be [2]. Grief before a close person's death has been conceptualized as anticipatory grief, a term that was first defined by Lindemann in light of the Freudian psychoanalytic theory. Anticipatory grief was seen as a form of 'grief work' before an actual loss, where the grieving person would gradually detach their bonds to the dying person [3]. This understanding of the concept of anticipatory grief has since been expanded, particularly in relation to palliative care, where family

\footnotetext{
* Correspondence: maja.holm@shh.se

${ }^{1}$ Department of Nursing Sciences, Sophiahemmet University, Box 5605, 114 86 Stockholm, Sweden

Full list of author information is available at the end of the article
}

caregivers may face a complex and stressful situation. They often spend a considerable amount of time caring for the patient [4] and their efforts are often indispensable to the health care system [5]. In order to promote efficient support, it has been stated that health care professionals should be attentive to anticipatory grief reactions in family caregivers [6] due to its potential consequences, such as emotional stress, loneliness, cognitive dysfunction, and social withdrawal [7]. Hence, there is a need to find methods to identify and measure anticipatory grief in family caregivers of patients who are in receipt of palliative care.

Of late, the concept of anticipatory grief has been discussed and sometimes renamed 'pre-death grief' or 'pre-loss grief' because it merely indicates the presence of grief symptoms before a person's death rather than anticipation of bereavement [8]. Other studies have indicated that there 
are some differences between these concepts, although they are often used interchangeably [9]. A recent review defined anticipatory grief as a multidimensional concept and a dynamic process [10]. Nuclear constructs of anticipatory grief have been described as anger, guilt, anxiety, irritability, sadness, feelings of loss, and decreased ability to function in performing usual tasks [11]. According to the original theory, anticipatory grief would improve bereavement outcomes because the grief work had already been commenced before the loss [3]. However, in later years, the theory of grief work has been questioned [12], and recent results indicate that experiencing high levels of anticipatory grief is associated with low preparedness for the loss, and additional problems in bereavement, such as complicated grief and post-loss depression [13, 14].

There are several different instruments constructed to measure anticipatory grief. Some of them are designed as 'pre-death' versions of instruments that are otherwise created to measure post-bereavement grief and these differentiate between normal or pathological grief. Examples are the pre-loss version of the PG-13 and the Pre-death Inventory of Complicated Grief [15, 16]. A recent review found six different instruments in 14 studies to measure anticipatory grief, and no more than three studies used the same instrument. Although they all aimed to measure caregivers' emotional status, there were substantial differences between the instruments. The authors of the review concluded that there is a lack of common understanding of the concept of anticipatory grief and a dearth of psychometric testing of the instruments used to measure the phenomenon [8].

One of the more widely used instruments designed to measure anticipatory grief is the Anticipatory Grief Scale (AGS), which was developed by Theut, Jordan, Ross, and Deutsch (1991). The AGS represents the major domains cited in the literature on grief. The items are constructed based on a combination of clinical experience and other instruments measuring grief, such as the Texas Revised Inventory of Grief (TRIG), which has been developed to measure grief in bereavement [17]. According to Theut et al., the AGS also investigates the reactions of grief, but before the loss. The AGS was originally developed as to be used on family caregivers of patients affected by dementia, however, the wording could be changed and used in other contexts, such as in cancer and palliative care [7, 18-20]. Although it has been used internationally, one important limitation is that the AGS has not been rigorously validated. Most important, even if AGS is constructed as a unidimensional measure of anticipatory grief, no previous study has evaluated the factor structure of the instrument, which is an important aspect of construct validity. Moreover, no previous study has evaluated the AGS conceptually for example by correltating the scores with similar and closely related concepts such as grief, anxiety or depression.
For further use in palliative care, the AGS also needs to be psychometrically evaluated when used with family caregivers of patients in palliative care. Hence, the aim of this study was to evaluate the psychometric properties, focusing on factor structure, of the Anticipatory Grief Scale in a sample of family caregivers in palliative care.

\section{Methods \\ Design}

This psychometric evaluation study was based on the baseline data from a previously conducted intervention trial, one that aimed to increase family caregivers' preparedness for providing palliative care. Details about the intervention study are presented elsewhere.

\section{Participants and procedure}

The study was conducted in the context of specialized palliative home care in a metropolitan area in Sweden. In all, 10 palliative care settings were involved. The palliative care provided by the home care settings included advanced symptom relief, palliative treatments and existential and emotional support. The settings were organized in multi-professional teams, including nurses, physicians, social workers and occupational and physical therapists. The inclusion criteria for the study were: being a family caregiver to a person in palliative care, over the age of 18 , and being able to understand the Swedish language. Designated health care professionals at each setting were responsible for the recruitment of family caregivers over a period of fifteen months in 2013 and 2014.

Patients were initially approached by the health care professionals and asked to agree that their family caregiver would be invited to participate in the study. They were also asked to consent to some information being taken from their medical records about their diagnosis and time of illness. If the patient agreed, the family caregiver was invited to participate in the study and was asked to complete a questionnaire, which included the AGS and additional questions about demographic background data. The questionnaire was returned to the research team by post.

\section{Instruments}

The questionnaire consisted of demographic questions (sex, age, social and economic situation and educational level) and self-rating scales, among them a Swedish version of the AGS. The questionnaire also included the Hospital Anxiety and Depression scale (HADS). In a later stage, after the patient's death, family caregivers answered the Texas Revised Inventory of Grief (TRIG). The two later instruments were used for construct validity purposes in the present study.

The AGS-scale consists of 27 items measuring anticipatory grief on a Likert scale, ranging from 1 (strongly disagree) to 5 (strongly agree). According to the 
constructor, the AGS items should be summed into a total score ranging from 27 to 135 with a higher score indicating higher levels of anticipatory grief. Eight items $(2,5,8,11,19,22,26,27)$ have a positive bearing and therefore must be reversed before the total score is calculated. No subscales or cut-off scores have been reported for the AGS. The original English version of the AGS has previously been translated into Swedish by a research group led by Associate Professor Grimby at Sahlgrenska Academy (unpublished). The group employed a standard procedure for translation and back-translation using two independent bilingual language experts. For the present study, several attempts have been made to communicate the validation process with the original authors, however, these attempts have been unsuccessful.

The Hospital Anxiety and Depression scale (HADS) has been developed to measure anxiety and depression over two subscales. The instrument consists of 14 items, of which seven measure anxiety and depression respectively. The questions are answered on a four point response scale ranging between 0 and 3 . The total score for each scale range between 0 and 21 where higher scores indicate more problems with anxiety or depression. The scale has shown satisfactory psychometric properties in different samples and language versions including the Swedish version [21].

The Texas Revised Inventory of Grief (TRIG) has been developed to measure the intensity of grief after bereavement. The instrument consists of two scales. TRIG I has eight items and involves thinking back to the time immediately after the loss (past behavior). TRIG II has 13 items and involves the bereaved person's current grief reactions (present feelings). The items are measured on a Likert-type scale between strongly agree (1) and strongly disagree (5). The total score ranges between 8 and 40 and 13-65 for TRIG I and TRIG II respectively. Lower scores indicate more intense grief reactions. The scale has shown satisfactory psychometric properties in family caregivers to patients in palliative care [22].

\section{Analysis}

Descriptive statistics were used to present demographic data and study variables.

All statistical analyses of the AGS was made after the scores of items with positive bearing had been reversed. Data quality was evaluated regarding the distribution of item and scale scores, and missing data patterns. Floor and ceiling effects for items, which refer to the proportions of participants with the lowest (floor) and highest (ceiling) possible scores, were evaluated using frequency distributions. A floor/ceiling effect of up to $20 \%$ was considered acceptable. The D'Agostino test, including skewness and kurtosis statistics, was conducted to evaluate whether the scale scores deviated significantly from the normal distribution. A normal distribution has skewness and kurtosis values close to 0 and 3, respectively, and a $p$-value $\geq 0.05$. A graphic examination of the scale score distribution was also conducted using normal probability plots (P-P and Q-Q plots). Missing data patterns were evaluated using percentages. Having up to $5 \%$ missing data was considered acceptable. Spearman's rank order correlation $\left(r_{s}\right)$ was used to evaluate associations between scale scores.

Homogeneity was evaluated with inter-item correlations and item-total correlations, using polychoric and polyserial correlations respectively (rho). Inter-item correlations between $0.15-0.85$ and item-total correlations $>0.3$ support homogeneity [23]. As calculations of polychoric correlations rely on an assumption of bivariate normality for the latent variables measured on a ordinal scale, a specific RMSEA test, developed and described by Jöreskog was applied to verify that there was no violation of this assumption. The RMSEA test for all pairs ranged between 0.000 and 0.091 which is below the recommended level of $<0.10$ [24]. Therfore, the polychoric correlations are probably unbiased and can be deemed as reliable coefficients.

The factor structure of the AGS was examined through exploratory factor analyses. The Kaiser-Meyer-Olkin test $(\mathrm{KMO}=0.84)$ and Bartlett's test $\left(\chi^{2}(351)=2270.3, p<\right.$ 0.001 ) supported the hypothesis that the data were suited for a factor analysis. As the items were treated as ordinal data, an unweighted least square (ULS) estimation method was used, and a polychoric correlation matrix was analyzed. A hot-deck multiple imputation was conducted for 14 participants who had incomplete data in the AGS. Using the same correlation matrix, a parallel analysis, based on optimal implementation and 500 replications, was conducted to identify the number of relevant factors to extract. To facilitate the interpretation of the factors, an ortogonal (varimax) rotaion was applied [25]. In the first step, a model that included all 27 items was conducted. A revised model was examined in a second step. Factor loadings, communality values $\left(h^{2}\right)$, the residual correlation matrix, and the Goodness of Fit Index (GFI) were all used to evaluate the models. To support model fit, factor loadings should be $>0.3$ on the actual factor, communality values should be $>0.3$, and GFI should be $>0.95$.

The internal consistency was evaluated using an ordinal variant of Cronbach's alpha, which is based on polychoric correlation rather than Pearson' correlations [26]. The interpretation should be regarded equally, i.e., alpha should be $>0.7$ [27]. For comparisons with previous studies, traditional alpha values were calculated.

Construct validity was evaluated through Spearman's rank correlations $\left(r_{\mathrm{s}}\right)$. Previous studies have shown that grief before death is associated with anxiety and depressive 
symptoms as well as grief after death. It was therefore hypothesized that AGS should correlate moderately to strongly with the HADS- anxiety, HADS depression and TRIG I and II $\left(r_{\mathrm{s}}=0.4-0.8\right)$. Because lower scores on TRIG indicates more grief, unlike AGS where lower scores indicates less grief, we expected negative correlations between these scales.

The FACTOR 10.3 (Rovira Virgili University, Tarragona, Spain) was used to perform the the factor analysis while LISREL 8.80 (Scientific Software International, Inc., Skokie, IL, USA) was used to test the assumptions of bivariate normality for polychoric correlations. All other analyses were conducted using the R 3.5.1 (The R Foundation for Statistical Computing, Vienna, Austria), including the PSYCH package. The level of statistical significance was overall set at $p<0.05$.

\section{Results}

\section{Characteristics of family caregivers}

In total, 270 family caregivers agreed to complete the AGS questionnaire. Most of them were women (68\%) and they had a mean age of $61.0(\mathrm{SD}=14.0)$ years. A majority $(75 \%)$ were family caregivers to a patient affected by cancer (Table 1).

\section{Item and scale score statistics}

Half of the items of the AGS either had a pronounced negative skew $(4,6,12,15,17,22)$ or a positive skew $(2,7,9,13,18,20,21,24)$ distribution, reflected by the median values and score distribution. Floor effects were demonstrated for 12 items and ceiling effects for 8 items. Problems with missing data were few between 0.3 and $1.5 \%$ across items (Table 2).

The original AGS total score followed a normal distribution, graphically (P-P and Q-Q plots) and statistically (skewness $=0.15$, kurtosis $=2.57, X^{2}(2)=3.62, p=0.164$ ) (Table 3).

\section{Homogeneity}

The inter-item correlations varied between rho -0.391 and 0.639 , with a mean rho of 0.182 . Two problems were identified. First, there were a substantial number of negative correlations $(n=65)$, which ranged between rho -0.001 and -0.391 (mean rho $=-0.116$ ). Second, 184 of 286 positive correlations were below rho $<0.3$. The positive inter-item correlations varied between rho 0.004 and 0.639 (mean rho $=0.250)$.

The item-total correlation revealed that 5 items had correlations below 0.3 (item 2, 5, 17, 19 \& 22), of which two had negative correlations (item 5 \& 22).

\section{Factor structure}

The parallel analysis carried out on the 27 items of the AGS advised that four factors should be retained from
Table 1 Characteristics of participants $(n=270)$

\begin{tabular}{|c|c|}
\hline Age, $m$ (SD) & $61.0(14.0)$ \\
\hline \multicolumn{2}{|l|}{ Gender, $n(\%)$} \\
\hline Men & 86 (31.8) \\
\hline Women & $184(68.2)$ \\
\hline \multicolumn{2}{|l|}{ Social status, $n(\%)$} \\
\hline Married/in partnership & $192(71.1)$ \\
\hline Unmarried & $78(28.9)$ \\
\hline \multicolumn{2}{|l|}{ Education, $n(\%)$} \\
\hline University & $118(43.7)$ \\
\hline Non-university & $152(56.3)$ \\
\hline \multicolumn{2}{|l|}{ Employment, $n$ (\%) } \\
\hline Employed & $134(49.6)$ \\
\hline Retired & $109(40.4)$ \\
\hline Other & $27(10.0)$ \\
\hline \multicolumn{2}{|l|}{ Living with patient, $n$ (\%) } \\
\hline Yes & $143(53.0)$ \\
\hline No & $127(47.0)$ \\
\hline \multicolumn{2}{|l|}{ Relation to patient, $n(\%)$} \\
\hline Spouse & $137(50.7)$ \\
\hline Adult child & $94(34.8)$ \\
\hline Other & $39(14.5)$ \\
\hline \multicolumn{2}{|l|}{ Patient diagnosis, $n$ (\%) } \\
\hline Cancer & $202(74.8)$ \\
\hline Other & 68 (25.2) \\
\hline
\end{tabular}

the AGS, explaining $52 \%$ of the total variance. The GFI of the model was 0.98 , indicating an excellent model fit. Eigenvalues of the four factors were all $>1$. Despite this, the four-factor model was problematic, because 10 of the 27 items loaded on more than one factor and two items did not load on any factor. Several items also demonstrated weak loadings in all four factors, and 6 had communality values below 0.3 (Table 4). As several of these problems were related to items with unclear directions (i.e. item 2, "I feel close to my relative who has incurable illness"), or were reversely scored, decision was made to evaluate a modified model by omitting these items. In total, 14 items were removed from the AGS.

The parallel analysis of the remaining 13 items in the AGS suggested a two-factor solution with adjusted eigenvalues of 4.68 for the first factor and 1.53 for the second factor, while subsequent factors were $<1$. These two factors explained $55 \%$ of the total variance. The factor loadings were all strong, ranging between $0.54-0.69$ for the first factor, and $0.63-0.82$ for the second factor. There were still two items that had double loadings (> 0.3 ), however the factor loadings were clearly pointing towards one of these two factors. The communalities of the items in the two-factor solution all exceeded 0.3 


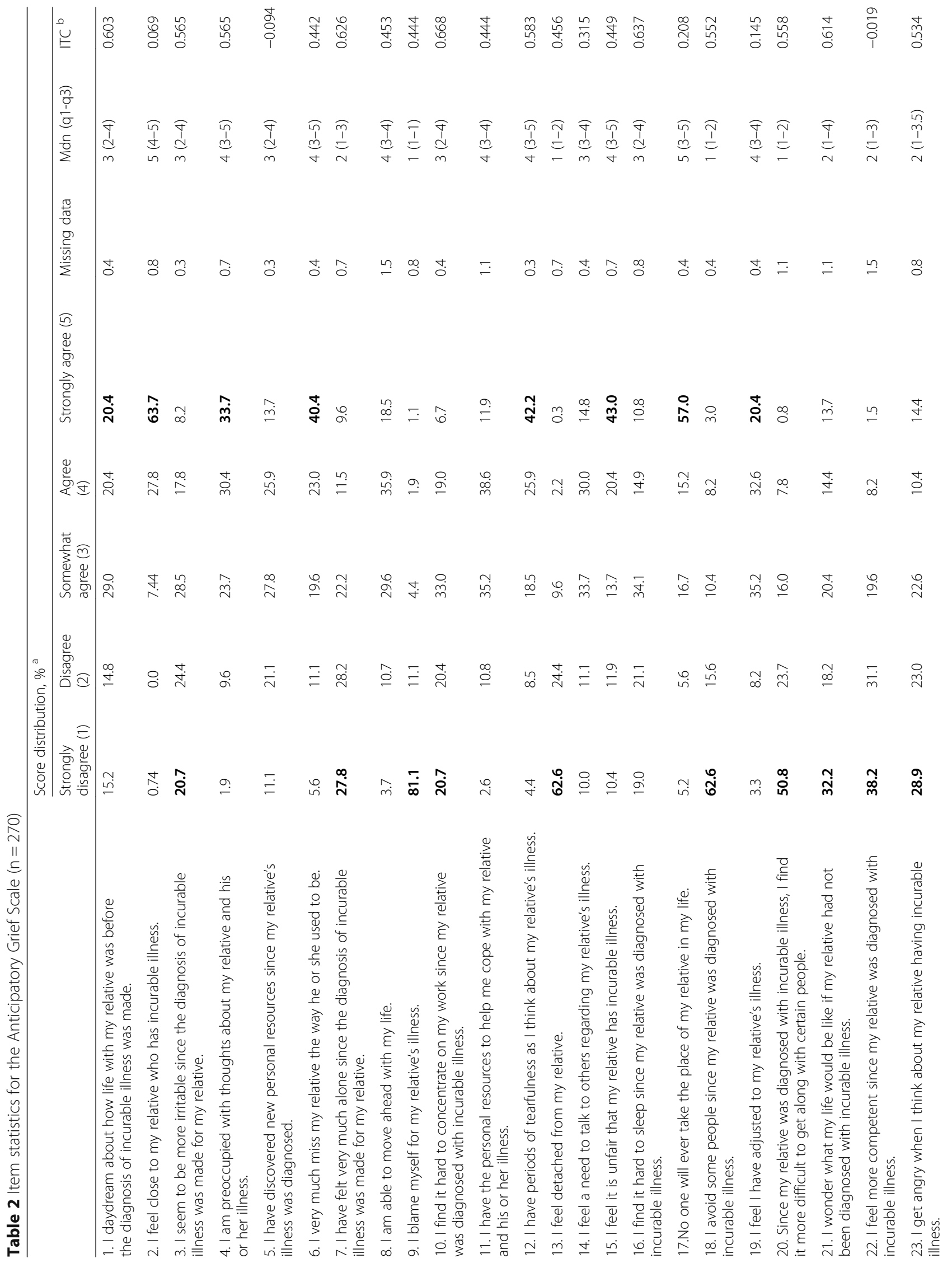




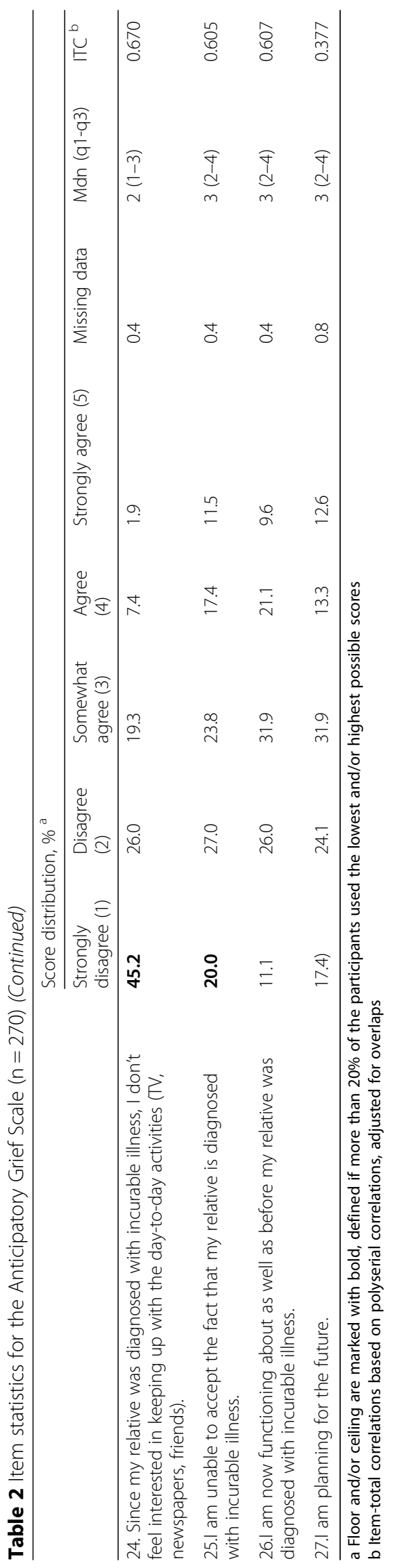


Table 3 Scale statistics for the Anticipatory Grief Scale

\begin{tabular}{|c|c|c|c|c|c|c|c|c|}
\hline \multirow[t]{2}{*}{ Factors/Scales } & \multicolumn{2}{|l|}{ Average score } & \multicolumn{3}{|c|}{ Score distribution $^{a}$} & \multicolumn{3}{|c|}{ Score correlation $^{b}$} \\
\hline & Mdn (q1-q3) & Range & Skewness & Kurtosis & $p$-value & Original AGS & Behavioral reactions & Emotional reactions \\
\hline Original AGS & $73(65-85)$ & $42-114$ & 0.15 & 2.57 & 0.164 & 1.00 & & \\
\hline Behavioral reactions & $19(16-25)$ & $8-35$ & 0.28 & 2.34 & 0.003 & $0.86^{* * *}$ & 1.00 & \\
\hline Emotional reactions & $15(11-19)$ & $5-25$ & -0.02 & 2.16 & $<0.001$ & $0.72^{* * *}$ & $0.43^{* * *}$ & 1.00 \\
\hline
\end{tabular}

${ }^{\text {a }}$ Tested with D'Agostino test

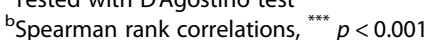

(Table 5). The GFI of the model was 0.98, indicating excellent model fit. The Kaiser-Meyer-Olkin test $(\mathrm{KMO}=$ $0.87)$ and Bartlett's test $\left(\chi^{2}(78)=1162.5, p<0.001\right)$ supported the hypothesis that the data were also suited for a factor analysis for this reduced model of 13 items.

Items $3,4,7,10,16,18,20$, and 24 were retained for the first factor, and items 1, 15, 21, 23, and 25 for the second factor. The content of the items was analyzed, and it was found that the first factor was associated with family caregivers' behavioral reactions to grief (e.g., "I avoid some people since my relative was diagnosed with incurable illness"). Hence this factor was named Behavioral reactions. The other factor seemed to be more attributed to the inner and emotional grief reactions (e.g., "I get angry when I think about my relative having incurable illness"). The second factor was named Emotional reactions.

\section{Scale score statistics of behavioral reactions and emotional reactions}

In contrast to the original AGS scale, both Behavioral reactions and Emotional reactions deviated significantly from normal distribution; the scores were not skewed but less peaked than expected for a normal distribution (skewness $=0.28$, kurtosis $=2.34, \chi^{2}(2)=11.69, p=0.003$ vs. skewness $=-0.02$, kurtosis $\left.=2.16, \chi^{2}(2)=19.17, p<0.001\right)$. Graphically, the Q-Q plot showed some deviations in the tails for both scales, while no deviations were detected in the P-P plot. The correlations between Behavioral reactions and Emotional reactions were moderate $\left(r_{s}=0.43\right)$ but both correlated strongly with the original AGS score that included all of the items ( $r_{s}=0.86$ vs. 0.72 , respectively).

\section{Construct validity}

Construct validity was supported for Behavioral reactions. The scale was substantially, but not too strongly correlated $(<0.8)$ with HADS-anxiety $\left(r_{\mathrm{s}}=0.70, p<\right.$ $0.001)$ and HADS-depression $\left(r_{\mathrm{s}}=0.65, p<0.001\right)$, aligning with the hypothesis that they measure related but different constructs. Behavioral reactions also correlated as expected with TRIG I - past behaviors $\left(r_{\mathrm{s}}=-0.55, p\right.$ $<0.001)$ and TRIG II - present feelings $\left(r_{\mathrm{s}}=-0.45, p<\right.$ 0.001). For Emotional reactions, construct validity was partly supported. The scale correlated weaker than expected with HADS-anxiety $\left(r_{\mathrm{s}}=0.36, p<0.001\right)$ and
HADS-depression $\left(r_{\mathrm{s}}=0.27, p<0.001\right)$. In contrast, the scale correlated as expected with TRIG I $\left(r_{\mathrm{s}}=-0.40, p<\right.$ $0.001)$ and TRIG II $\left(r_{\mathrm{s}}=-0.48, p<0.001\right)$.

\section{Internal consistency}

The ordinal alpha for the AGS-scale including all 27 items was 0.91, indicating excellent internal consistency. The traditional Cronbach's alpha coefficient showed corresponding findings $(\alpha=0.87)$. The ordinal alpha for the two factors generated from the final factor model was 0.83 and 0.84 for the Behavioral reactions and Emotional reactions, respectively, indicating that internal consistency was excellent. The corresponding values, measured with the traditional Cronbach's alpha, were 0.82 and 0.82 , respectively (Table 5 ).

\section{Discussion}

According to our best knowledge, this is the first study that has validated the Anticipatory Grief Scale. The results from the exploratory factor analyses in a sample of 270 family caregivers indicate that, although it has been used in several studies, the original version of the instrument is flawed with regards to its psychometric quality in the context of palliative care. Based on the results of the present study, a modified version of AGS with 13 items over two subscales was suggested which appears to be promising regarding the quality, homogeneity, factor structure and internal consistency of the data. This modified version of the AGS will hereafter be named AGS-13 and include items 3, 4, 7, 10, 16, 18, 20 and 24 to measure Behavioral reactions, and item 1, 15, 21, 23 and 25 to measure Emotional reactions.

The item score distribution of the original AGS was skewed, with ceiling and floor effects for two-thirds of the items. However, this was not considered to be a serious problem, because the total score of the AGS was normally distributed and all the response options were used and there was a variation in the score distribution. Expressed ceiling and floor effects could influence sensitivity and responsiveness [28]. However, the results indicated no such problems in the original AGS or in the two subscales of the AGS-13, Behavioral reactions and Emotional reactions. Unlike the original scale, the two subscales both deviated statistically from a normal 
Table 4 Results from the exploratory factor analysis (unweighted least square) of the original Anticipatory Grief Scale $(n=270)$

\begin{tabular}{|c|c|c|c|c|c|}
\hline \multirow[t]{2}{*}{ Item numbers } & \multicolumn{4}{|c|}{ Factors and factor loadings ${ }^{a}$} & \multirow[t]{2}{*}{$\mathrm{h} 2^{\mathrm{b}}$} \\
\hline & l & $\|$ & III & IV & \\
\hline 1 & 0.648 & & 0.302 & & 0.523 \\
\hline 2 & & & & 0.685 & 0.600 \\
\hline 3 & & 0.339 & 0.426 & & 0.343 \\
\hline 4 & & & 0.619 & & 0.537 \\
\hline 5 & & & & 0.563 & 0.389 \\
\hline 6 & 0.417 & & 0.346 & & 0.331 \\
\hline 7 & & 0.392 & 0.473 & & 0.443 \\
\hline 8 & & & 0.414 & & 0.242 \\
\hline 9 & & 0.588 & & & 0.440 \\
\hline 10 & & 0.375 & 0.677 & & 0.626 \\
\hline 11 & & & 0.400 & 0.437 & 0.391 \\
\hline 12 & 0.445 & & 0.480 & & 0.583 \\
\hline 13 & & 0.502 & & 0.545 & 0.581 \\
\hline 14 & & & 0.310 & & 0.130 \\
\hline 15 & 0.805 & & & & 0.666 \\
\hline 16 & & & 0.552 & & 0.471 \\
\hline 17 & & & & & 0.240 \\
\hline 18 & & 0.604 & & & 0.456 \\
\hline 19 & & & & 0.447 & 0.221 \\
\hline 20 & & 0.731 & & & 0.613 \\
\hline 21 & 0.612 & 0.326 & & & 0.521 \\
\hline 22 & & & & & 0.241 \\
\hline 23 & 0.776 & & & & 0.656 \\
\hline 24 & & 0.523 & 0.379 & & 0.498 \\
\hline 25 & 0.707 & & & & 0.558 \\
\hline 26 & & & 0.663 & & 0.534 \\
\hline 27 & & & 0.445 & & 0.230 \\
\hline Explained variance, (\%) & 26.3 & 12.1 & 7.5 & 6.5 & \\
\hline Cum. Variance, (\%) & 26.3 & 38.4 & 45.9 & 52.4 & \\
\hline
\end{tabular}

${ }^{\mathrm{a}}$ Factor loadings below 0.3 are omitted

${ }^{\mathrm{b} C o m m u n a l i t y ~ v a l u e s}$

distribution, but graphically, they were close to a normal distribution.

Few missing values were identified which indicates that the instrument was easy to complete. The item with the highest rate of missing values was number 22 which had a rate of $1.5 \%$ missing values, which is decidedly below the level of acceptance of $5 \%$. Item 22 was removed from the AGS-13. In the AGS-13, there were few problems with missing values as none of the items exceeded $0.8 \%$ in missing data.

The original AGS demonstrated low homogeneity for several items according to inter-item and item-total
Table 5 Results from the exploratory factor analysis (unweighted least square estimation with varimax rotation of the factors) of the modified Anticipatory Grief Scale $(n=270)$

\begin{tabular}{|c|c|c|c|}
\hline \multirow[t]{2}{*}{ Item numbers } & \multicolumn{2}{|c|}{ Factors and factor loadings ${ }^{a}$} & \multirow[t]{2}{*}{$\mathrm{h} 2^{\mathrm{b}}$} \\
\hline & I & $\|$ & \\
\hline \multicolumn{4}{|l|}{ Behavioral reactions } \\
\hline 3 & 0.548 & & 0.343 \\
\hline 4 & 0.537 & & 0.342 \\
\hline 7 & 0.582 & & 0.392 \\
\hline 10 & 0.755 & & 0.587 \\
\hline 16 & 0.578 & 0.314 & 0.433 \\
\hline 18 & 0.603 & & 0.377 \\
\hline 20 & 0.691 & & 0.479 \\
\hline 24 & 0.639 & & 0.480 \\
\hline \multicolumn{4}{|l|}{ Emotional reactions } \\
\hline 1 & & 0.654 & 0.488 \\
\hline 15 & & 0.816 & 0.668 \\
\hline 21 & 0.350 & 0.631 & 0.520 \\
\hline 23 & & 0.727 & 0.553 \\
\hline 25 & & 0.702 & 0.549 \\
\hline Explained variance (\%) & 40.0 & 15.3 & \\
\hline Cum. Variance (\%) & 40.0 & 55.3 & \\
\hline Ordinal alpha & 0.83 & 0.84 & \\
\hline
\end{tabular}

${ }^{\mathrm{b} C o m m u n a l i t y ~ v a l u e s}$

correlations. Several items had unclear directions, which could explain the low homogeneity. A further explanation could be that the scale consists of several dimensions, which was also supported by the factor analysis. The results, based on the original AGS, with 27 items, suggested that 4 factors should be retained. However, these factors were inconsistent and difficult to describe conceptually. A possible explanation could be that the AGS includes items with both positive and negative bearing (i.e., reversely scored), something that could lead to greater inconsistencies in the responses, although it has also been speculated that it could reduce acquiescence bias [29]. The poor fit of the original scale to the factor model could also possibly be explained by the fact that the AGS was not originally developed for family caregivers in palliative care. Although the author has stated that the instrument could be used for other diagnoses [30]), it seems as though some items were developed specifically to be used on family caregivers in dementia care (i.e., I feel detached from my relative). Family caregivers with experiences from different care contexts may interpret response options in different ways and their conceptualization of the measured construct could also differ [31]. Unfortunately, there are no other evaluation studies on the AGS and, therefore, it is 
possible that the original scale also has the same measurement problems in other groups.

In the AGS-13, the 8 items with positive bearing (i.e., reversely scored) and items that were related specifically to family caregivers of patients with dementia were not included. The exclusion of the positive items could be questioned, because they might capture aspects of grief that the remaining, negatively loaded items may not [32]. However, after thorough, consideriations, it was interpreted that the remaining items covered important aspects of the content from the removed items, e.g. feelings towards daily life and mood changes. Earlier research has found that including a few items with reversed directions than the majority of items in the general scale increases the risk of misreading the reversed items, because the person is being asked to shift a mental gear in processing the information [33]. Aligning with the results of this study, reversed items often have lower item-total correlation and they could generally fit less well to factor models [34]. This could invalidate a proposed scale, which could, in fact, be valid and reliable [29]. Items were also removed from the AGS due to weak loadings or unclear directions.

Apart from performing statistical analyses, it is also necessary to review the conceptualization of anticipatory grief over time [31]. According to the constructor, the AGS scale is consistent with existing theoretical and empirical evidence concerning anticipatory grief. However, the scale dates back to 1991 and, of late, the concept has been reconsidered [8]. Anticipatory grief was originally seen as being a part of the total grief work that the family caregiver passed through. In later research, the hypothesis of grief work has been reconsidered and more recent conceptual models of grief describe it as a dual process of both loss and restoration [32]. The two subscales extracted from the AGS-13 were only moderately correlated, which suggests that they would measure two separate, but related constructs.

Construct validity was demonstrated for the AGS-13 subscale Behavioral reactions and agreed with our hypothesis that it would be moderately to strongly associated with anxiety, depression and post-death grief. Even though Emotional reactions correlated moderately with the TRIG-scales, its correlation with anxiety and depression was weak and construct validity was only partly supported. It could be that Behavioral reactions is simply a conceptually stronger subscale. However, it could also be that the concepts measured in Emotional reactions (yearning anger, feeling a lack of fairness and inacceptance of the condition) represent a disorder that is essentially different from anxiety and depression.

Reducing the number of items and creating a modified version of the AGS could make the scale more useful in clinical practice as there is a need for instruments that are not only psychometrically sound but also brief and easy to use, especially due to the vulnerability of respondents in palliative care [35]. The two scales of the AGS-13 had somewhat lower internal consistency compared with the original scale. This outcome was expected, as the ordinal alpha, as well as the traditional Cronbach's alpha, will increase with number of items [36]. Therefore, it can be argued that the internal consistency was even better in the AGS-13, because the two scales included only 8 and 5 item each, compared with the original 27 -item version. Further, the alpha level still indicated excellent internal consistency after the instrument was modified.

\section{Strengths and limitations}

This study used exploratory factor analyses because the factor structure of the original AGS was unknown. Hence, the suggested factor model needs to be confirmed in future studies. In total, 14 items were excluded from the AGS, and it is possible that some items were conceptually important in capturing the phenomenon of anticipatory grief. It would seem as though the AGS-13 with its subscales $\mathrm{Be}$ havioral reactions and Emotional reactions measure dimensions of the loss-oriented form of grief rather than restoration-oriented grief. This includes activities and emotions dealing with separation from an attachment figure, and could be compared to the traditional understanding of grief work. The 8 items with positive bearing that were removed from the scale could possibly have captured the more restoration-oriented form of grief [32] and it is also possible that some of the removed items were relevant, not only for dementia, but also in palliative care. The strengths of this validation include that the statistical tests were adapted to ordinal data. Polychoric correlations, a technique to estimate the bivariate correlation between two latent normally distributed continuous variables measured using an ordinal scale [37], are commonly recommended for ordinal data as parametric methods commonly will underestimate the population correlation [38]. The estimation method, ULS with a polychoric correlation matrix, provides accurate factor loadings, and less variable parameter estimates, as well as more precise standard errors compared to other methods ([39] \{Li, 2016 \#7389). The factor analysis was performed in a sample of 270 family caregivers, which could be considered adequate for the study with a variable ratio of 10:1 [40]. However, a general rule of thumb for exploratory factor analysis is that weak data (low communalities and crossloadings) demands a greater sample [41]. With the AGS-13, items with weak data were removed and the sample size was significantly improved (variable ratio 20:1). However, this validation study needs to be replicated with a larger sample, and there is also a need to validate whether the AGS-13 is invariant across different language versions over time and across different groups, for example with regard to age, sex, and ethnicity and also for family caregivers of patients with different diagnoses in palliative care. It would also be valuable 
to identify cutoff scores for clinical importance for the AGS-13 which could be used by health care professionals to identify family caregivers in need of support during palliative caregiving.

\section{Conclusions}

In conclusion, it was reasonable to create a modified version of the AGS, as using the original version with its statistical weaknesses could be considered unethical, and it would be inappropriate to overburden family caregivers with unnecessary questions. The AGS-13 version contains fewer items and could be used more easily to capture behavioral and emotional reactions of grief. The AGS-13 might have the potential to be used in future studies of anticipatory grief among family caregivers in palliative care. However, the factor structure needs to be confirmed in further studies.

\section{Abbreviations}

AGS: Anticipatory Grief Scale; GFI: Goodness of Fit Index; KMO: Kaiser-MeyerOlkin; TRIG: Texas Revised Inventory of Grief; ULS: Unweighted Least Square

\section{Acknowledgements}

We acknowledge the work of the original authors of the AGS, Susan K. Theut, Linda Jordan, Louis A. Ross and Stephen I. Deutsch and thank them for their important contribution to the field of grief research.

\section{Funding}

This study received financial support from the Swedish Cancer Society.

\section{Availability of data and materials}

Please contact the authors for data requests.

\section{Authors' contributions}

$\mathrm{MH}, \mathrm{AA}$ and $\mathrm{K} \AA$ were involved in study designing, data collection, analysis and drafting the manuscript. CJF and JÖ were involved in study design and revision of the manuscript. All authors have approved the final draft of the manuscript.

\section{Ethics approval and consent to participate}

The study was approved by a regional ethical review board in Stockholm, Sweden (No. 2012/377). Written informed consent was obtained both by patients and family caregivers before participants were enrolled in the study.

\section{Consent for publication}

The manuscript contains no personally identifiable information of the participants.

\section{Competing interests}

The authors declare that they have no competing interests.

\section{Publisher's Note}

Springer Nature remains neutral with regard to jurisdictional claims in published maps and institutional affiliations.

\section{Author details}

'Department of Nursing Sciences, Sophiahemmet University, Box 5605, 114 86 Stockholm, Sweden. ${ }^{2}$ Department of Health Care Sciences, Ersta Sköndal University College, Box 11189, 10061 Stockholm, Sweden. ${ }^{3}$ Capio Geriatrics, Palliative care unit, Dalen hospital, Åstorpsringen 6, 121 87, Stockholm,

Sweden. ${ }^{4}$ Department of Clinical Science and the Institute for Palliative Care, Lund University, Scheelevägen 2, 22381 Lund, Sweden. Institute of Health and Care Sciences, Sahlgrenska Academy, University of Gothenburg, Box 457, 40530 Gothenburg, Sweden. ${ }^{6}$ Centre for Person-Centred Care, University of Gothenburg, Arvid Wallgrens backe 1, 41346 Gothenburg, Sweden. ${ }^{7}$ Faculty of Health and Life Sciences, Linnaeus University, 39182 Kalmar, Sweden. ${ }^{8}$ The Reserch Section, Region Kalmar County, Lasarettsvägen 1, 39244 Kalmar, Sweden.

Received: 1 November 2017 Accepted: 25 February 2019

Published online: 05 March 2019

\section{References}

1. Jordan A, Litz B. Prolonged grief disorder: diagnostic, assessment, and treatment considerations. Prof Psychol Res Pract. 2014;45:180-7.

2. Parkes, C.M.P, H Bereavement: Studies of grief in adult life, Fourth Edition., New York: Routledge 2010

3. Lindemann E. Symptomatology and management of acute grief. Am J Psychiatry. 1944;151(6 Suppl):155-60.

4. Rowland $\mathrm{C}$, et al. The contributions of family care-givers at end of life: a national post-bereavement census survey of cancer carers' hours of care and expenditures. Palliat Med. 2017;31(4):346-55.

5. Grande $\mathrm{G}$, et al. Supporting lay carers in end of life care: current gaps and future priorities. Palliat Med. 2009:23(4):339-44.

6. Moon PJ. Anticipatory grief: a mere concept? Am J Hosp Palliat Care. 2016; 33(5):417-20.

7. Johansson AK, Grimby A. Anticipatory grief among close relatives of patients in hospice and palliative wards. Am J Hosp Palliat Care. 2012;29(2):134-8.

8. Nielsen MK, et al. Do we need to change our understanding of anticipatory grief in caregivers? A systematic review of caregiver studies during end-oflife caregiving and bereavement. Clin Psychol Rev. 2016:44:75-93.

9. Lindauer A, Harvath TA. Pre-death grief in the context of dementia caregiving: a concept analysis. J Adv Nurs. 2014;70(10):2196-207.

10. Coelho A, Barbosa A. Family anticipatory grief: an integrative literature review. Am J Hosp Palliat Care. 2016;34(8)774-785.

11. Rando TA. Anticipatory grief: the term is a misnomer but the phenomenon exists. J Palliat Care. 1988;4(1-2):70-3

12. Stroebe W, Schut H, Stroebe MS. Grief work, disclosure and counseling: do they help the bereaved? Clin Psychol Rev. 2005;25(4):395-414.

13. Nielsen MK, et al. Pre-loss grief in family caregivers during end-of-life cancer care: a nationwide population-based cohort study. Psychooncology. 2017.

14. Nielsen MK, et al. Predictors of complicated grief and depression in bereaved caregivers: a Nationwide prospective cohort study. J Pain Symptom Manag. 2017;53(3):540-50.

15. Tomarken A, et al. Factors of complicated grief pre-death in caregivers of cancer patients. Psychooncology. 2008;17(2):105-11.

16. Thomas K, et al. Risk factors for developing prolonged grief during bereavement in family carers of cancer patients in palliative care: a longitudinal study. J Pain Symptom Manag. 2014;47(3):531-41.

17. Faschingbauer T. Texas revised inventory of grief manual. Houston, Texas: Honeycomb Publishing Co; 1981.

18. Liu NC, Lai EY. Find a way out: bereavement support in Taiwan hospice. Support Care Cancer. 2006;14(1):4-10.

19. Johansson UE, Grimby A. Anticipatory grief among close relatives of patients with Parkinson's disease. Behavioral sciences. 2014;3(5):179-84.

20. Burke $L A$, et al. Risk factors for anticipatory grief in family members of terminally ill veterans receiving palliative care services. J Soc Work End Life Palliat Care. 2015;11(3-4):244-66.

21. Lisspers J, Nygren A, Soderman E. Hospital anxiety and depression scale (HAD): some psychometric data for a Swedish sample. Acta Psychiatr Scand. 1997;96(4):281-6.

22. Holm M, et al. Psychometric evaluation of the Texas revised inventory of grief in a sample of bereaved family caregivers. Res Nurs Health. 2018;41(5):480-8.

23. Clark LA, Watson D. Constructing validity: basic issues in objective scale development. Psychol Assess. 1995;7(3):309-19.

24. Jöreskog KG. Structural equation modelling with ordinal varaibles using LISREL. Skokie, IL: Scientific Software International; 2005.

25. Pett MA, Lackey NR, Sullivan JJ. Making sense of factor analysis: the use of factor analysis for instrument development in healt. Thousand Oaks, CA: Sage; 2003.

26. Gadermann AM. G.M., Zumbo BD, Estimating ordinal reliability for Likert-type and ordinal item response data: a conceptual, empirical, and practical guide. . Practical assessment. Research \& Evaluation. 2012;17(3):1-13.

27. Nunally, J.C.B, I. Psychometric theory. $3^{\text {rd }}$ edition, New York, Ny: McGraw-Hill 1994.

28. Fayers P, Machin D. Quality of life: the assessment, analysis, and interpretation of patient-reported outcomes. 2nd ed. Chichester: Wiley; 2007 
29. Solis Salazar M. The dilemma of combining positive and negative items in scales. Psicothema. 2015;27(2):192-200.

30. Theut SK, et al. Caregiver's anticipatory grief in dementia: a pilot study. Int J Aging Hum Dev. 1991;33(2):113-8.

31. Sawatzky R, et al. Modern perspectives of measurement validation emphasize justification of inferences based on patient-reported outcome scores: seventh paper in a series on patient reported outcomes. J Clin Epidemiol. 2016.

32. Stroebe M, Schut $\mathrm{H}$. The dual process model of coping with bereavement: rationale and description. Death Stud. 1999;23(3):197-224.

33. Roszkowski MJS. M., Shifting gears: consequences of including two negatively worded items in the middle of a positively worded questionnaire. Assess Eval High Educ. 2010;35(1):117-34.

34. Weijters B, Baumgartner H. Misresponse to reversed and negated items in surveys: a review. J Mark Res. 2012;49(5):737-47.

35. Hudson PL, et al. A systematic review of instruments related to family caregivers of palliative care patients. Palliat Med. 2010;24(7):656-68.

36. Streiner D, Norman G. Health measurement scales: a practical guide to their development and use. 2nd ed. Oxford: Oxford University Press; 2015

37. Olsson U. Maximum likelihood estimation of the polychoric correlation coefficient. Psykometrika. 1979;44:443-4.

38. Brown AB. Confirmatory factor analysis for applied research. 2nd ed. New York: The Guilford Press; 2015.

39. Forero CG, Maydeu-Olivares A, Gallardo-Pujol D. Factor analysis with ordinal indicators: a Monte Carlo study comparing DWLS and ULS estimation. Structural Equation Modeling-a Multidisciplinary Journal. 2009:16(4):625-41.

40. Hair J, RL ART, Black WC. Multivariate data analysis. 4th ed. New Jersey: Prentice-Hall Inc; 1995.

41. Costello A, Osborne J. Best practices in exploratory factor analysis: four recommendations for getting the Most from your analysis. Pract Assess Res Eval. 2005;10(7).

Ready to submit your research? Choose BMC and benefit from:

- fast, convenient online submission

- thorough peer review by experienced researchers in your field

- rapid publication on acceptance

- support for research data, including large and complex data types

- gold Open Access which fosters wider collaboration and increased citations

- maximum visibility for your research: over $100 \mathrm{M}$ website views per year

At $\mathrm{BMC}$, research is always in progress.

Learn more biomedcentral.com/submissions 